\title{
The serum metabolic profiles of different Barcelona stages hepatocellular carcinoma associated with hepatitis B virus
}

\author{
LEI ZHANG ${ }^{1,2^{*}}$, GUANG-YE WU ${ }^{3 *}$, YU-JING WU ${ }^{1,4^{*}}$ and SHU-YE LIU ${ }^{1,4}$ \\ ${ }^{1}$ Tianjin Key Laboratory of Artificial Cell, Artificial Cell Engineering Technology Research Center of \\ Public Health Ministry, Tianjin Third Central Hospital, Tianjin 300170; ${ }^{2}$ Chemical Engineering Institute, \\ Tianjin Univeristy, Tianjin 300072; ${ }^{3}$ Department of Clinical Laboratory, The Second Affiliated Hospital of \\ Tianjin University of Traditional Chinese Medicine, Tianjin 300150; \\ ${ }^{4}$ Clinical Laboratory Department, Tianjin Third Central Hospital, Tianjin 300170, P.R. China
}

Received January 6, 2016; Accepted May 25, 2017

DOI: $10.3892 / \mathrm{ol} .2017 .7393$

\begin{abstract}
The present study aimed to explore the characteristic ions distinguishing different Barcelona stages in patients with hepatitis B virus (HBV)-associated hepatocellular carcinoma (HCC) using the ultra-performance liquid chromatography-mass spectrometry (UPLC-MS) platform, and to evaluate their value in diagnosing and monitoring the progress of HCC. The serum was sampled from 20 healthy volunteers, 20 patients with $\mathrm{HBV}$-induced cirrhosis and 75 patients with $\mathrm{HBV}$-associated HCC of different BCLC stages. Samples were all examined using UPLC-MS. Principal components analysis (PCA) and the orthogonal partial least squares discriminant analysis (OPLS-DA) model were constructed to determine potential biomarkers. Then, the independent sample-nonparametric test was used to perform the final screening for ion identification. Receiver operating characteristic (ROC) curve analysis was used to evaluate the diagnostic value of these ions. Serum metabolomic PCA and OPLS-DA models were established to diagnose different BCLC stages of HCC associated with HBV, with OPLS-DA model parameters ( $\mathrm{R} 2 \mathrm{X}=67.2 \%, \mathrm{R} 2 \mathrm{Y}=82 \%, \mathrm{Q} 2 \mathrm{Y}=61.1 \%)$. A total of 20 metabolites with statistically significant differences among groups were identified, primarily including amino acids, bile acid, fatty acid and phosphatidate. The area under the curve (AUC) of LysoPC [18:2 (9Z,12Z)], LysoPC (P-16:0), asparaginyl-proline and vaccenic acid in the comparison between HCC and cirrhosis were all increased compared with
\end{abstract}

Correspondence to: Professor Shu-Ye Liu, Clinical Laboratory Department, Tianjin Third Central Hospital, 83 Jintang Road, Tianjin 300170, P.R. China

E-mail: yujing_wu5@163.com

${ }^{*}$ Contributed equally

Key words: hepatitis B virus, hepatocellular carcinoma, metabonomics, Barcelona stage, ultra-performance liquid chromatography-mass spectrometry that of AFP, indicating a more improved diagnosis ability. Furthermore, the AUC of L-aspartyl-4-phosphate and LysoPC [20:5 $(5 Z, 8 Z, 11 Z, 14 Z, 17 Z)]$ in the stage A vs. B comparison were increased compared with that of AFP, but were decreased in the comparison between stage B and C. The present study succeeded in screening metabolic ions that reflect the progress of HCC with high diagnostic value. Thus, the identified ions may serve a role in clinically diagnosing $\mathrm{HBV}$-associated $\mathrm{HCC}$ and monitoring the development of the disease.

\section{Introduction}

The morbidity rate of hepatocellular carcinoma (HCC) is the fifth highest among all types of malignant tumor and the third leading cause of cancer-associated mortality worldwide (1). Chronic hepatitis B virus (HBV) infection accounts for $\sim 50 \%$ of total cases and almost all childhood HCC cases (2). Thus, the identification of biomarkers to diagnose and indicate the stage of disease in patients with HBV-associated HCC is warranted. The Barcelona Clinic Liver Cancer (BCLC) staging system performs well in guiding therapeutic decision making for $\mathrm{HCC}$, and takes into consideration the neoplasm, liver function and general condition of patients combined with treatment principles (3). Thus, in the present study, BCLC was chosen as the standard system for different stages of HCC.

Metabolomics studies small molecules (molecular weight, $<1,800 \mathrm{Da}$ ), which define the metabolic status of a biological system. It has been used extensively to identify biomarkers for HCC. The cyclic adenosine monophosphate, glutamine, and short- and medium-chain acylcarnitines were reported as differential metabolites of cirrhosis, and HCC (4). Zhou et al (5) reported that the proinflammatory precursor arachidonic acid level is increased significantly in patients with HCC compared with those with cirrhosis and healthy controls. These studies indicate that metabolomics may be a promising diagnostic tool for HCC.

In the present study, using the basic principles and technology of metabolomics, the changes of small molecular metabolites were analyzed, and monitored in patients with HBV-associated HCC with different BCLC stages. 


\section{Materials and methods}

Chemicals and instruments. All solvents were high-performance liquid chromatography (HPLC) grade and used without modification. Formic acid and acetonitrile (ACN) were obtained from Sigma-Aldrich; Merck KGaA (Darmstadt, Germany). Distilled water was produced using a Milli-Q Reagent Water system (EMD Millipore, Billerica, MA, USA). All standard [L-phenylalanine, glycocholic acid and lysophosphatidylcholine (LysoPC), 14:0] preparations were purchased from Sigma-Aldrich; Merck KGaA. Ultra HPLC was performed using a Thermo Fisher Accela system (Thermo Fisher Scientific, Inc., Waltham, MA, USA). Mass spectrometry (MS) was performed on a Thermo Fisher LTQ Orbitrap XL hybrid mass spectrometer (Thermo Fisher Scientific, Inc.). Other equipment included a Multifuge X1R high-speed centrifuge (Thermo Fisher Scientific, Inc.).

Patients. In the present study, 75 patients with $\mathrm{HBV}$-associated HCC, 20 patients with HBV-induced cirrhosis and 20 healthy volunteers at the Tianjin Third Central Hospital (Tianjin, China) between November 2013 and January 2015 were enrolled. The following inclusion criteria for patients with $\mathrm{HCC}$ was maintained: i) Immunologic test indicates HBV surface antigen-positive; ii) HCC was confirmed by pathological diagnosis or multi-slice computer tomography scan, and/or dynamic contrast-enhanced magnetic resonance imaging; iii) patients did not receive any previous antitumor therapy; iv) patients without secondary liver cancer or combined with other system tumors; and v) patients without complications of gastrointestinal bleeding or tumor rupture hemorrhage. All 75 patients with HCC according to the Barcelona liver cancer staging were divided to three groups: Stage A, early stage patients who could be treated radically (including stage A1-A4, but excluding stage $A 0, n=26$ ); stage $B$, middle stage patients who could be treated by arterial chemo embolization $(n=23)$; and stage $\mathrm{C}$ group, late stage patients who could only accept symptomatic treatment $(n=26)$. Furthermore, the inclusion criteria for patients with $\mathrm{HBV}$-induced cirrhosis were as follows: i) HBV surface antigen-positive; ii) all 20 patients with liver cirrhosis were at the compensated stage, child-pugh scores A-B; iii) cirrhosis was diagnosed by abdominal ultrasonography and transient elastography; and iv) patients without any other malignant diseases or complications of hemorrhage. In addition, no severe infection was detected and parenteral nutrition was used for all patients. The dietary requirements of patients were managed by the Nutrition Department of the Tianjin Third Central Hospital to a relatively uniform standard, as a result, exogenous dietary influence on metabolic profiling was limited to the lowest level.

Serum sample collection. The clinical data of patients, including sex, age, prothrombin time, and albumin, globulin, bilirubin, aspartate aminotransferase, alanine aminotransferase and $\alpha$-fetoprotein (AFP) levels were collected from the medical records (Table I). No significant differences were identified in age (result of one-way analysis of variance: $\mathrm{F}=0.395 ; \mathrm{P}=0.757$ ) or sex (result of $\chi^{2}$ test: $\chi^{2}=3.419 ; \mathrm{P}=0.490$ ), ensuring comparability of data between groups. A total of $4 \mathrm{ml}$ fasting venous blood samples were collected from subjects in the early morning and placed in the separation gel tube. Blood serum was packed and storing for analysis at $-80^{\circ} \mathrm{C}$ following centrifugation at 2,280 x g for $10 \mathrm{~min}$. Blood samples and clinical data were collected according to the Helsinki declarations under the consent of patients and approved by the Ethics Committee of Tianjin Third Central Hospital.

Pretreatment. The specimens were thawed at room temperature, methanol was added to the serum samples according to the ratio 1:3 (200:600 $\mu 1)$. Subsequently, samples were agitated for $30 \mathrm{sec}$ using an oscillator, then left to stand for $5 \mathrm{~min}$. The samples were centrifuged at $10,000 \mathrm{x} \mathrm{g}$ for $30 \mathrm{~min}$ at $4^{\circ} \mathrm{C}$. The supernatant was dried using vacuum concentration at $45^{\circ} \mathrm{C}$ for $3 \mathrm{~h}$. A total of $400 \mathrm{ml}$ solvent was prepared for dissolving the dry powder. The mixture was agitated for $30 \mathrm{sec}$. Lastly, samples were filtered through a membrane with $0.22 \mu \mathrm{m}$ apertures.

Sample analysis. Chromatography conditions were as follows: Chromatography was performed using a Thermo Fisher Accela equipped with a binary solvent delivery manager and a sample manager. The analytical column was a Thermo Hypersil GOLD C18 reversed phase column (2.1 mm ID x $50 \mathrm{~mm}$, $1.9 \mu \mathrm{m}$; Thermo Fisher Scientific, Inc.).

The mobile phase consisted of the following: phase A $0.1 \%$ formic acid (volume ratio), $1 \mathrm{ml}$ of formic acid was added to a 1 liter bottle of HPLC-grade water; phase B 95\% ACN and $0.1 \%$ formic acid, $950 \mathrm{ml} \mathrm{ACN}, 50 \mathrm{ml} \mathrm{HPLC}$-grade water and $1 \mathrm{ml}$ formic acid were combined.

Chromatographic separation was performed isocratically within $15 \mathrm{~min}$ and the injection volume was $10 \mu \mathrm{l}$. The flow rate was set at $200 \mu \mathrm{l} / \mathrm{min}$. The sample manager and column oven temperature were set at 4 , and $20^{\circ} \mathrm{C}$, respectively. The chromatographic elution gradient was initialized at $5 \%$ phase $\mathrm{B}$ and held for $3 \mathrm{~min}$. In consecutive $10 \mathrm{~min}$ periods, phase B was gradually increased to $50 \%$, and then a rapid increase in phase B to $95 \%$ was completed within $3 \mathrm{~min}$. After $4 \mathrm{~min}$ of maintaining a high volume of organic phase gradient, phase $\mathrm{B}$ was immediately reduced to $5 \%$ and this elution gradient was used to balance the analytical column for the final $4 \mathrm{~min}$.

MS was performed using a Thermo Fisher LTQ Orbitrap XL hybrid mass spectrometer, operating in the positive ion mode with an ion source voltage of $4.5 \mathrm{kV}$, a capillary voltage of $30 \mathrm{~V}$, cone voltage of $150 \mathrm{~V}$, desolvation temperature of $350^{\circ} \mathrm{C}$, sheath gas flow of $30 \mathrm{arb}$. and assistant gas flow of 5 arb. (99.999\% nitrogen). Data were collected over $15 \mathrm{~min}$ in the centroid mode over the mass range $50-1,000 \mathrm{~m} / \mathrm{z}$. The MS resolution was set at 100,000 full width half maximum and the calibration standards were provided by Thermo Fisher Scientific, Inc. (caffeine, Ultramark 1621 and MRFA). MS/MS analysis was performed with collision-induced dissociation with collision energy 35 (normalization collision energy) and the collision gas was $99.999 \%$ helium.

There were 20 quality control (QC) samples throughout the test (equal volume mixture of each analyzed sample). Prior to analysis, eight QC samples were analyzed continuously and the remaining 12 QC samples were inserted into the sequence after every 10 samples were analyzed. The sequence of samples was randomly generated by the excel function prior to and following sample analysis (including QC), and 
Table I. Basic characteristics of patients.

\begin{tabular}{|c|c|c|c|c|c|}
\hline \multirow[b]{2}{*}{ Characteristics } & \multicolumn{3}{|c|}{ HCC group } & \multirow[b]{2}{*}{$\begin{array}{l}\text { LC group } \\
(\mathrm{n}=20)\end{array}$} & \multirow[b]{2}{*}{$\begin{array}{l}\text { Health control } \\
\qquad(\mathrm{n}=20)\end{array}$} \\
\hline & $\begin{array}{l}\text { Early stage } \\
\quad(n=26)\end{array}$ & $\begin{array}{l}\text { Middle stage } \\
\quad(\mathrm{n}=23)\end{array}$ & $\begin{array}{l}\text { Late stage } \\
\qquad(n=26)\end{array}$ & & \\
\hline Sex (male/female) & $17 / 9$ & $19 / 4$ & $22 / 4$ & $15 / 5$ & $16 / 4$ \\
\hline Age (years) & $55.64 \pm 8.49$ & $55.28 \pm 6.26$ & $54.84 \pm 9.91$ & $53.05 \pm 8.92$ & $52.73 \pm 6.27$ \\
\hline PT (sec) & $\begin{array}{c}14.15 \\
13.43-15.40\end{array}$ & $\begin{array}{c}14.00 \\
13.60-14.90\end{array}$ & $\begin{array}{c}14.80 \\
14.20-15.40\end{array}$ & $\begin{array}{c}18.00^{\mathrm{a}} \\
15.00-19.95\end{array}$ & $13.22 \pm 0.88$ \\
\hline Albumin (g/l) & $\begin{array}{l}42.10 \pm 3.42 \\
40.35-44.55\end{array}$ & $\begin{array}{c}39.90 \\
34.55-43.18\end{array}$ & $\begin{array}{c}36.10^{\mathrm{b}} \\
30.88-37.50\end{array}$ & $\begin{array}{c}34.15^{\mathrm{a}} \\
26.88-38.53\end{array}$ & $\begin{array}{c}47.70 \\
45.4-49.40\end{array}$ \\
\hline Globulin (g/l) & $\begin{array}{c}27.20 \\
24.30-30.30\end{array}$ & $\begin{array}{c}30.40 \\
24.90-33.80\end{array}$ & $\begin{array}{c}31.10 \\
27.45-37.10\end{array}$ & $\begin{array}{c}29.60 \\
27.90-42.13\end{array}$ & $\begin{array}{c}26.84 \\
23.96-29.84\end{array}$ \\
\hline $\operatorname{ALT}(\mathrm{U} / \mathrm{l})$ & $\begin{array}{c}34.00 \\
20.50-45.00\end{array}$ & $\begin{array}{c}47.00 \\
28.00-80.00\end{array}$ & $\begin{array}{c}49.00 \\
38.25-63.75\end{array}$ & $\begin{array}{c}59.00 \\
29.75-307.50\end{array}$ & $\begin{array}{c}18.00 \\
15.00-21.00\end{array}$ \\
\hline AST (U/l) & $\begin{array}{c}31.50 \\
22.75-37.50\end{array}$ & $\begin{array}{c}61.50^{\mathrm{a}} \\
28.50-85.50\end{array}$ & $\begin{array}{c}108.00 \\
54.00-161.00\end{array}$ & $\begin{array}{c}48.00^{\mathrm{a}} \\
45.00-314.00\end{array}$ & $\begin{array}{c}20.00 \\
17.00-24.00\end{array}$ \\
\hline $\mathrm{BIL}(\mu \mathrm{mol} / \mathrm{l})$ & $\begin{array}{c}16.00 \\
12.70-19.55\end{array}$ & $\begin{array}{c}13.65 \\
10.38 \sim 26.20\end{array}$ & $\begin{array}{c}26.90^{\mathrm{b}} \\
19.63-39.70\end{array}$ & $\begin{array}{c}41.10^{\mathrm{a}} \\
20.40-92.35\end{array}$ & $\begin{array}{c}8.57 \\
6.49-11.20\end{array}$ \\
\hline $\operatorname{AFP}(\mathrm{ng} / \mathrm{ml})$ & $\begin{array}{c}15.29 \\
5.75-110.00\end{array}$ & $\begin{array}{c}631.80^{\mathrm{a}} \\
34.94-1210.00\end{array}$ & $\begin{array}{c}1210.00 \\
650.28-1210.00\end{array}$ & $\begin{array}{c}14.86 \\
6.56-91.37\end{array}$ & - \\
\hline
\end{tabular}

${ }^{a} \mathrm{P}<0.05$ compared to that of early stage HCC group; ${ }^{\mathrm{b}} \mathrm{P}<0.05$ compared to middle stage HCC group. HCC, hepatocellular carcinoma; PT, prothrombin time; ALT, alanine aminotransferase; AFP, $\alpha$-fetoprotein; AST, aspartate aminotransferase; BIL, bilirubin; LC, liver cirrhosis .

cross-contamination was avoided by inserting a blank between adjacent samples. The whole experiment lasted 3,960 min.

Data treatment. MZmine software (version 2.0) was used to analyze the original data derived from the UPLC-MS platform (6). Detailed standards were as follows: i) The peak signal-to-noise ratio $(\mathrm{S} / \mathrm{N})>30$; ii) retention time interval in the range of $\pm 0.1 \mathrm{~min}$; iii) $\mathrm{m} / \mathrm{z}$ deviation $< \pm 0.02$. The mode establishing method with SIMCA-P+ software (version 12.0.1; Umetrics; Sartorius AG, Göttingen, Germany) was used to filter the variations, thus forming the orthogonal partial least-squares discriminant analysis (OPLS-DA) model $(7,8)$. Potential biomarkers were preliminarily screened using visual methods in the model, namely $\mathrm{S}$ figure, shared and unique structure plot figure, variable influence on projection (VIP) and confidence interval (CI) (9). Detailed standards were VIP $>1$ and 0 not in CI.

SPSS software (version 17.0; SPSS, Inc., Chicago, IL, USA) was used to evaluate the statistical significance of differences of the variances between different groups. A one-way analysis of variance with post hoc least significant difference correction was applied for multiple comparisons between quantitative data. Statistical differences were analyzed by a two-tailed Mann-Whitney U test. A $\chi^{2}$ test was used to compare the sex ratio between multiple groups. $\mathrm{P}<0.05$ was considered to indicate a statistically significant difference. The receiver operating characteristic (ROC) curves were generated and the corresponding area under the curve (AUC) was calculated. A comparison was conduct between potential biomarkers identified, and AFP, which is the most widely used biomarker for HCC.

The metabolites were identified according to the following steps: i) The Human Metabolome DataBase was searched with an accurate $\mathrm{m} / \mathrm{z}$ value (10); ii) two stage mass spectrometry of characteristic ions were obtained using MS/MS scanning, which were combined with the physical and chemical characteristics of metabolites to make a preliminary judgment; iii) at least two independent and orthogonal biochemical data, including the use of accurate mass, and retention time, were used for identification according to Guideline for metabolite identification (11); and iv) the retention time and MS were compared with those of a standard product for further identification. For those without reliable standard products, identification was based on specific physical and chemical properties.

\section{Results}

Data pretreatment and quality control analysis. The total ion chromatogram of the early stage group (stage A), middle stage group (stage B) and late stage group (stage C) acquired by the UPLC-MS platform are presented in Fig. 1. Following pretreatment and standardization using MZmine 2.0 software, 489 integral peaks following extraction ion chromatography were detected in QC samples, and 515 peaks in test samples.

The stability of the UPLC-MS system was adequately assessed through analysis of QC samples during the entire experimental period. Through principal component analysis 


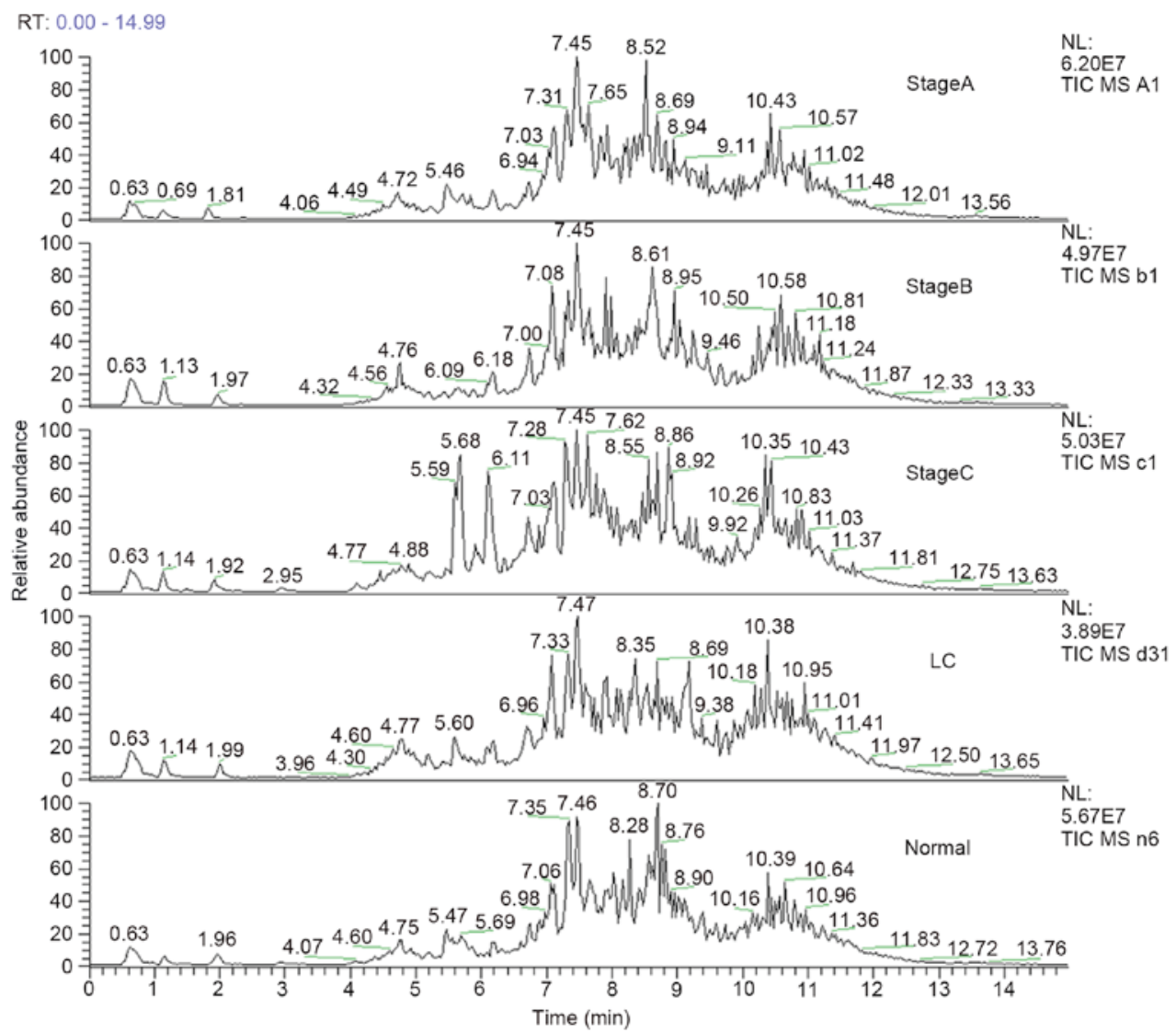

Figure 1. Total ion chromatogram of tissue metabolic profiling. This was the total ion chromatogram of one single sample chosen randomly (stage A, early period group BCLC stage A; stage B, middle period group BCLC stage B; stage C, later period group BCLC stage C; LC, liver cirrhosis group; normal, healthy control group).

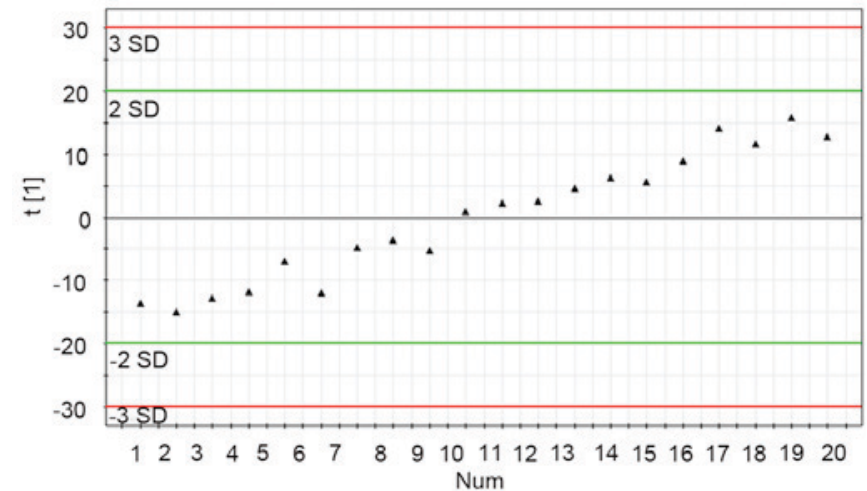

Figure 2. Score plot of principal component [t(1)] for quality control principal component analysis model. each point represents a quality control sample.

(PCA) of 20 data sets of QC samples, a PCA model with one principal component was established (Fig. 2). Fig. 2 demonstrates the score plot of QC sample sequences vs. the first principal component (the most influential factor which varied with time). From the QC principal component score plot, it was demonstrated that the UPLS-MS system was stable following the first eight continuous QC injections. In the test sample sequence, a QC sample was inserted after every 10 test samples to evaluate the stability of the system during the entire analytical process. The results revealed that the detection system was stable throughout the experiment following the first eight QC samples injected (no outliers exceeding \pm 2 standard of deviation were detected in the QC samples). According to a previous study (12), the QC standard was set as follows: i) Ion peaks were defined as reliable peaks when their intensity was in the range of $\pm 30 \%$ average ion intensity; ii) a QC sample qualified if its 70\% ion peaks were reliable; and iii) experimental data were accepted only when $60 \%$ QC was qualified. In the present experiment, all the QC samples (reliable ion peaks distributed among 80.5-89.3\%) inserted into the test sample sequence qualified and the qualified ratio was $100 \%$, which indicated that the analytical results were valid.

Ability of the metabolic profile to distinguish disease states. First a PCA model having nine predictive principal components $\left(\mathrm{R}_{2} \mathrm{X}=47.2 \% ; \mathrm{Q}_{2}=26 \%\right)$ was established (Fig. $3 \mathrm{~A}$ ), but the PCA model distinguishing healthy people from the other groups, failed to differentiate cirrhosis and different stages of HCC. Then, the OPLS-DA model with four predictive principal components and four orthogonal principal components $\left(\mathrm{R}_{2} \mathrm{X}=65.5 \%, \mathrm{R}_{2} \mathrm{Y}=75.6 \%\right.$; $\left.\mathrm{Q}_{2} \mathrm{Y}=57.4 \%\right)$ was established (Fig. 3B). In the OPLS-DA model, the normal and late stage groups demonstrated significant clustering tendency in the first predictive principal component, while the cirrhosis group demonstrated significant clustering tendency in the second predictive principal component. In addition, another OPLS-DA model was established (Fig. 3C) with three HCC groups and the normal group, which possessed three predictive principal components and seven orthogonal principal components 

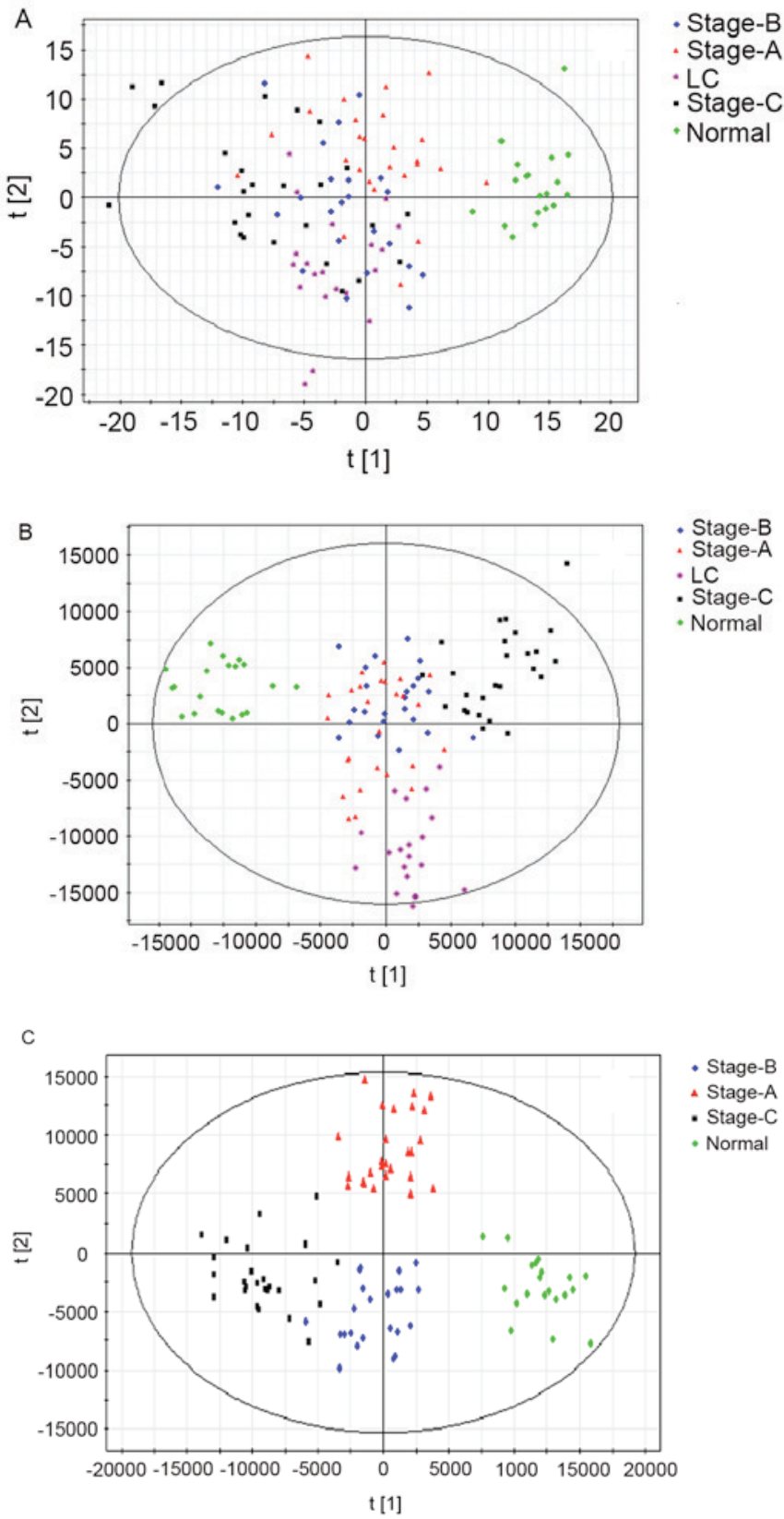

Figure 3. Ability of metabolic profiling to distinguish disease. (A) Score plot of the first two components $[\mathrm{t}(1) / \mathrm{t}(2)]$ of the all serum samples metabolic profiling principal component analysis model. (B) Score plot of all serum samples metabolic profiling orthogonal partial least squares discriminant analysis model. (C) Score plot of three HCC groups and normal group samples metabolic profiling orthogonal partial least squares discriminan analysis model. Each point in the figure represents one sample. Stage A, early period group BCLC stage A; stage B, middle period group BCLC stage $\mathrm{B}$ stage C, later period group BCLC stage C; LC, liver cirrhosis group; normal, healthy control group.

$\left(\mathrm{R}_{2} \mathrm{X}=67.2 \%, \mathrm{R}_{2} \mathrm{Y}=82 \% ; \mathrm{Q}_{2} \mathrm{Y}=61.1 \%\right)$. In this model, the score plot of the first predictive principal component and second predictive principal component revealed significant clustering tendency. Different HCC stages and healthy controls were well distinguished in this model.

Selection and identification of characteristic metabolites. Using the aforementioned screening steps, 20 characteristic metabolites distinguishing early stage HCC and late stage
HCC were selected (Table II). A total of six metabolites were able to differentiate liver cirrhosis from HCC, in addition, seven metabolites could differentiate health controls from HCC. A total of nine metabolites were able to distinguish early stage HCC from other stages, and eight metabolites could distinguish late stage HCC from other stages.

ROC analysis. A total of six metabolites were able to differentiate liver cirrhosis from each HCC stage group, namely LysoPC [18:2 (9Z,12Z)], LysoPC (P-16:0), asparaginyl-proline, vaccenic acid, L-aspartyl-4-phosphate and LysoPC [20:5 (5Z,8Z,11Z,14Z,17Z)]. The levels of first three metabolites were significantly higher in the HCC group compared with in the cirrhosis group, with AUC values of $0.826,0.822$ and 0.820 , respectively (Table III). The AUCs of these metabolites differentiating between stage A HCC and cirrhosis were $0.688,0.858$ and 0.975 , respectively. Furthermore, vaccenic acid was significantly decreased in each HCC stage compared with in the cirrhosis group. AUCs of vaccenic acid distinguishing between HCC with cirrhosis and stage A HCC with cirrhosis were 0.798 and 0.869 , respectively. AUC values of AFP distinguishing between HCC from cirrhosis and stage A HCC with cirrhosis were 0.737 and 0.519 , respectively. All differences were identified to be statistically significant $(\mathrm{P}<0.05)$. The serum level of L-Aspartyl-4-phosphate in patients with HCC was significantly decreased with the progression of disease. The serum level of LysoPC [20:5 (5Z,8Z,11Z,14Z,17Z)] was significantly increased with the progression of HCC. AUC values are listed in Table III.

\section{Discussion}

HBV can cause liver inflammation, resulting in liver cell proliferation. The gene fragment of HBV can be combined with the gene of liver cells, and the HBV protein interacts with the DNA in the liver cells, resulting in HCC (13). However, the infection of HBV in a liver carcinoma cell is influenced by various factors. The mechanism is so complex that the optimal way to improve the survival of patients with $\mathrm{HCC}$ is to diagnose and treat the disease at an early stage.

In the present retrospective study, the healthy control, HCC and LC group demonstrated significant clustering tendency in the PCA model, indicating that liver damage, tumor and other hepatitis B-associated disease factors were major factors in the generation of model clustering. However, the LC and HCC group were not well distinguished. The probable reason was that the majority of patients with HCC exhibited basic cirrhosis disease. Although no significant clustering tendency was identified between the three different HCC stages, a clustering tendency was observed with disease progression.

The first OPLS-DA model distinguished the healthy control and late stage HCC group well from the other groups, but no significant clustering tendency was identified between the LC group, early, and middle stage HCC groups. The probable reasons are as follows: i) Among the BCLC groups, tumor metastasis or invasion of vascular lymph nodes was required in the diagnosis of late stage HCC, which could be detected definitely. The boundaries were clear in this group. However, HCC in the early and middle stages was diagnosed by the tumor 
Table II. Identification and difference of characteristic metabolites.

\begin{tabular}{|c|c|c|c|c|c|c|c|c|c|}
\hline \multirow[b]{2}{*}{ No. } & \multirow[b]{2}{*}{$\mathrm{m} / \mathrm{z}$} & \multirow[b]{2}{*}{$\begin{array}{l}\text { Retention } \\
\text { time (min) }\end{array}$} & \multirow[b]{2}{*}{ Metabolite } & \multirow[b]{2}{*}{ Adduct $^{b}$} & \multicolumn{5}{|c|}{ Content $^{\mathrm{c}}$} \\
\hline & & & & & $\begin{array}{l}\text { LC vs. } \\
\text { HCC }\end{array}$ & $\begin{array}{l}\text { Normal vs. } \\
\text { HCC }\end{array}$ & $\begin{array}{l}\text { Stage A vs. } \\
\text { stage B }\end{array}$ & $\begin{array}{c}\text { Stage B vs. } \\
\text { stage C }\end{array}$ & $\begin{array}{l}\text { Stage A vs. } \\
\text { stage C }\end{array}$ \\
\hline 1 & 520.344 & 7.087 & LysoPC [18:2 (9Z,12Z)] & $\mathrm{M}+\mathrm{H}[1+]$ & $\downarrow^{*}$ & - & $\downarrow^{*}$ & - & $\downarrow^{*}$ \\
\hline 2 & 568.34 & 7.004 & $\begin{array}{l}\text { LysoPC [22:6 (4Z,7Z, } \\
10 Z, 13 Z, 16 Z, 19 Z)]\end{array}$ & $\mathrm{M}+\mathrm{H}[1+]$ & - & - & - & $*$ & $\uparrow *$ \\
\hline 3 & 188.069 & 1.928 & L-phenylalanine ${ }^{\mathrm{a}}$ & $\mathrm{M}+\mathrm{Na}[1+]$ & - & - & - & $\uparrow$ & $\uparrow$ \\
\hline 4 & 468.304 & 6.697 & LysoPC $(14: 0)^{\mathrm{a}}$ & $\mathrm{M}+\mathrm{H}[1+]$ & - & - & $\uparrow$ & - & $\uparrow$ \\
\hline 5 & 337.271 & 6.965 & Pregnanetriol & $\mathrm{M}+\mathrm{H}[1+]$ & - & - & $\uparrow$ & - & $\uparrow$ \\
\hline 6 & 214.004 & 0.702 & L-Aspartyl-4-phosphate & $\mathrm{M}+\mathrm{H}[1+]$ & $\uparrow$ & $\downarrow$ & $\downarrow$ & $\downarrow$ & $\downarrow$ \\
\hline 7 & 248.122 & 5.690 & Threoninyl- $\gamma$-glutamate & $\mathrm{M}+\mathrm{H}[1+]$ & - & $\uparrow$ & $\uparrow$ & - & $\uparrow$ \\
\hline 8 & 530.355 & 7.971 & LysoPC (P-18:0) & $\mathrm{M}+\mathrm{Na}[1+]$ & - & $\downarrow$ & - & $\downarrow$ & $\downarrow$ \\
\hline 9 & 480.342 & 8.103 & LysoPC (P-16:0) & $\mathrm{M}+\mathrm{H}[1+]$ & $\downarrow$ & - & - & $\uparrow$ & $\uparrow$ \\
\hline 10 & 270.142 & 5.694 & Threoninyl-lysine & $\mathrm{M}+\mathrm{Na}[1+]$ & - & $\uparrow$ & $\uparrow$ & - & $\uparrow$ \\
\hline 11 & 283.261 & 7.851 & Vaccenic acid & $\mathrm{M}+\mathrm{H}[1+]$ & $\uparrow$ & - & $\downarrow$ & - & $\downarrow$ \\
\hline 12 & 274.091 & 4.042 & Deoxyadenosine & $\mathrm{M}+\mathrm{Na}[1+]$ & - & - & - & $\uparrow$ & $\uparrow$ \\
\hline 13 & 230.115 & 5.687 & Asparaginyl-proline & $\mathrm{M}+\mathrm{H}[1+]$ & $\downarrow$ & $\uparrow$ & $\uparrow$ & - & $\uparrow$ \\
\hline 14 & 412.28 & 5.591 & LPA (P-16:0e/0:0) & $\mathrm{M}+\mathrm{NH} 4[1+]$ & - & $\downarrow$ & $\downarrow$ & - & $\downarrow$ \\
\hline 15 & 176.062 & 0.936 & Guanidinosuccinic acid & $\mathrm{M}+\mathrm{H}[1+]$ & - & - & $\uparrow$ & - & $\uparrow$ \\
\hline 16 & 542.322 & 6.935 & $\begin{array}{l}\text { LysoPC (20:5 (5Z,8Z, } \\
11 Z, 14 Z, 17 Z))\end{array}$ & $\mathrm{M}+\mathrm{H}[1+]$ & - & - & $\uparrow$ & $\uparrow$ & $\uparrow$ \\
\hline 17 & 488.294 & 5.589 & Glycocholic acid ${ }^{\mathrm{a}}$ & $\mathrm{M}+\mathrm{Na}[1+]$ & - & $\downarrow$ & - & $\downarrow$ & $\downarrow$ \\
\hline 18 & 370.292 & 6.721 & cis-5-tetradecenoylcarnitine & $\mathrm{M}+\mathrm{H}[1+]$ & - & - & - & $\downarrow$ & $\downarrow$ \\
\hline 19 & 641.434 & 8.630 & $\begin{array}{l}\text { Ganglioside GM3 } \\
(\mathrm{d} 18: 0 / 25: 0)\end{array}$ & $\mathrm{M}+2 \mathrm{H}[2+]$ & - & - & - & $\downarrow$ & $\downarrow$ \\
\hline 20 & 284.292 & 6.728 & Octadecanamide & $\mathrm{M}+\mathrm{H}[1+]$ & $\uparrow$ & - & - & - & - \\
\hline
\end{tabular}

${ }^{a}$ Metabolites identified by standard comparison; ${ }^{b}$ Ionospheric models of mass spectrometry cationic scanning; ${ }^{c}$ Comparison of characteristic metabolites' integral peak area in the five groups. Arrows indicate $\mathrm{P}<0.05$ compared between samples using Mann-Whitney test. LysoPC, lysophosphatidylcholine.

Table III. Areas under receiver operating characteristic curves of HCC grading biomarkers.

\begin{tabular}{|c|c|c|c|c|c|c|}
\hline \multirow[b]{2}{*}{ Metabolite } & \multicolumn{2}{|c|}{ AUC } & \multicolumn{2}{|c|}{ P-value } & \multicolumn{2}{|c|}{$95 \% \mathrm{CI}$} \\
\hline & $\mathrm{A} / \mathrm{B}$ & $\mathrm{B} / \mathrm{C}$ & $\mathrm{A} / \mathrm{B}$ & $\mathrm{B} / \mathrm{C}$ & $\mathrm{A} / \mathrm{B}$ & $\mathrm{B} / \mathrm{C}$ \\
\hline L-Aspartyl-4-phosphate & 0.768 & 0.639 & 0.002 & 0.104 & $0.624-0.911$ & $0.477-0.802$ \\
\hline LysoPC [20:5 (5Z,8Z,11Z,14Z,17Z)] & 0.828 & 0.673 & 0.000 & 0.032 & $0.700-0.956$ & $0.499-0.812$ \\
\hline AFP & 0.647 & 0.704 & 0.079 & 0.017 & $0.511-0.842$ & $0.550-0.858$ \\
\hline
\end{tabular}

A-C refers to HCC stages A-C, respectively. HCC, hepatocelluar carcinoma; AUC, area under the curve; CI, confidence interval; AFP, $\alpha$-fetoprotein.

size and number, resulting in unavoidable cross phenomenon between those two groups. As a result, those two groups were not distinguished as well as the late stage group. ii) Patients in the HCC group exhibited varying degrees of hepatic fibrosis. In the present study, the majority of liver damage in patients with LC was in the compensatory phase, which was similar to that of patients in the early and middle stage HCC groups. However, the majority of patients in the late stage HCC group exhibited severe cirrhosis, and the difference was significant compared with patients in the cirrhosis group. Thus, the aforementioned clustering phenomenon appeared in the models. However, on the whole, a trend of clustering was observed with progression of the disease. Furthermore, in the second principal component, early, middle and late stage HCC groups, and the cirrhosis group were well distinguished. This indicated that the OPLS-DA model constructed in the current study had 
good interpretation and prediction ability for HBV-associated HCC disease progression. Following removal of the cirrhosis group, the second OPLS-DA model was consistent with the aforementioned model, and HCC in early and middle stage groups could be separated in the second principal component.

According to the metabolites identification methods, 20 metabolites associated with HCC were identified, primarily lysophosphatidyl choline, amino acids, cholylglycine and vaccenic acid. These metabolites are potential biomarkers for the development of HBV-associated HCC. In the differential diagnosis of cirrhosis and early stage HCC, the AUCs of LysoPC [18:2 (9Z,12Z)], LysoPC (P-16:0), asparaginyl-proline and vaccenic acid were increased compared with that of AFP. This indicates that these four metabolites performed better compared with AFP in the diagnosis HCC, particularly in early stage HCC.

Tan et al (14) compared the metabolites of patients with HCC and cirrhosis, and healthy controls. It was demonstrated that LysoPC (P-16:0) and LysoPC (22:5) (combined sensitivity and combined specificity, 80.5 and $80.1 \%$, respectively) performed better compared with AFP (sensitivity and specificity, 53 and $64 \%$, respectively) in the diagnosis of patients with HCC with $<2 \mathrm{~cm}$ diameter tumors (13). Cao et al (15) revealed that the levels of LPC (P-18:0) and LPC (P-16:0) were higher in patients with $\mathrm{HCC}$ compared that of healthy controls, consistent with the current study. In addition, the results of the present study demonstrated that levels of certain metabolites, including LysoPC [20:5 (5Z,8Z,11Z,14Z,17Z)] and LysoPC (14:0), decreased with the development of HCC. The downregulation of lysoPCs may primarily result from rapid membrane PC turnover during liver injury or malignant regeneration (16). The possible mechanism associating this observation is that HCC development severely damages liver function, thus reducing the number of enzymes available to produce PC. As a result, LPC is produced. Another possible reason is that the defensive system of body is weakened, thus PC synthesis is reduced (17). The AUCs of L-aspartyl-4-phosphate and LysoPC [20:5 (5Z,8Z,11Z,14Z,17Z)] were higher compared with that of AFP when differentiating between early, and middle stage HCC. While the AUC of AFP was increased compared with that of the two in differentiating between middle and late stage HCC. L-Aspartyl-4-phosphate and LysoPC [20:5 (5Z,8Z,11Z,14Z,17Z)] combined with AFP can significantly improve the capability to predict HCC stage.

In conclusion, a metabolic profiling model was successfully established, revealing that metabolomics is a promising tool to identify characteristic metabolites in the serum to diagnose different HCC stages. A total of four characteristic metabolites were identified to differentiate between HCC and liver cirrhosis. In addition, two metabolites performed well in distinguishing between different HCC stages. Additional large sample size studies are warranted to confirm the clinical value of these metabolites.

\section{Acknowledgements}

The present study was supported by the General Project of Application Infrastructure and Cutting-Edge Technology Research Programs, Tianjin (grant no. 13JCYBJC22100), and the Program Project of Health and Family Planning Commission Technology Fund, Tianjin (grant no. 2014KY01).

\section{References}

1. Mittal S and El-Serag HB: Epidemiology of hepatocellular carcinoma: Consider the population. J Clin Gastroenterol 47 (Suppl): S2-S6, 2013.

2. El-Serag HB: Epidemiology of viral hepatitis and hepatocellular carcinoma. Gastroenterology 142: 1264-1273, 2012.

3. Chapiro J and Geschwind JF: Hepatocellular carcinoma: Have we finally found the ultimate staging system for HCC? Nat Rev Gastroenterol Hepatol 11: 334-336, 2014.

4. Shao Y, Zhu B, Zheng R, Zhao X, Yin P, Lu X, Jiao B, Xu G and Yao Z: Development of urinary pseudotargeted LC-MS-based metabolomics method and its application in hepatocellular carcinoma biomarker discovery. J Proteome Res 14: 906-916, 2015.

5. Zhou L, Ding L, Yin P, Lu X, Wang X, Niu J, Gao P and Xu G: Serum metabolic profiling study of hepatocellular carcinoma infected with hepatitis B or hepatitis $\mathrm{C}$ virus by using liquid chromatography-mass spectrometry. J Proteome Res 11: 5433-5442, 2012.

6. Steinfath M, Groth D, Lisec J and Selbig J: Metabolite profile analysis: From raw data to regression and classification. Physiol Plant 132: 150-161, 2008.

7. Pham-Tuan H, Kaskavelis L, Daykin CA and Janssen HG: Method development in high-performance liquid chromatography for high-throughput profiling and metabonomic studies of biofluid samples. J Chromatogr B Analyt Technol Biomed Life Sci 789: 283-301, 2003.

8. Katajamaa M and Oresic M: Processing methods for differential analysis of LC/MS profile data. BMC Bioinformatics 6: 179, 2005.

9. Yin P, Wan D, Zhao C, Chen J, Zhao X, Wang W, Lu X, Yang S, $\mathrm{Gu} \mathrm{J}$ and $\mathrm{Xu} \mathrm{G}$ : A metabonomic study of hepatitis B-induced liver cirrhosis and hepatocellular carcinoma by using RP-LC and HILIC coupled with mass spectrometry. Mol Biosyst 5: 868-876, 2009.

10. Chen J, Zhao X, Fritsche J, Yin P, Schmitt-Kopplin P, Wang W, Lu X, Häring HU, Schleicher ED, Lehmann R and Xu G: Practical approach for the identification and isomer elucidation of biomarkers detected in a metabonomic study for the discovery of individuals at risk for diabetes by integrating the chromatographic and mass spectrometric information. Anal Chem 80: 1280-1289, 2008.

11. Sumner LW, Amberg A, Barrett D, Beale MH, Beger R, Daykin CA, Fan TW, Fiehn O, Goodacre R, Griffin JL, et al: Proposed minimum reporting standards for chemical analysis Chemical Analysis Working Group (CAWG) Metabolomics Standards Initiative (MSI). Metabolomics 3: 211-221, 2007.

12. Dunn WB, Broadhurst D, Brown M, Baker PN, Redman CW, Kenny LC and Kell DB: Metabolic profiling of serum using ultra performance liquid chromatography and the LTQ-Orbitrap mass spectrometry system. J Chromatogr B Analyt Technol Biomed Life Sci 871: 288-298, 2008.

13. Funk ML, Rosenberg DM and Lok AS: World-wide epidemiology of HBeAg-negative chronic hepatitis B and associated precore and core promoter variants. J Viral Hepat 9: 52-61, 2002.

14. Tan Y, Yin P, Tang L, Xing W, Huang Q, Cao D, Zhao X, Wang W, Lu X, Xu Z, et al: Metabolomics study of stepwise hepatocarcinogenesis from the model rats to patients: Potential biomarkers effective for small hepatocellular carcinoma diagnosis. Mol Cell Proteomics 11: M111.010694, 2012.

15. CaoH,Huang H,Xu W, ChenD, YuJ,LiJ andLi L:Fecalmetabolome profiling of liver cirrhosis and hepatocellular carcinoma patients by ultra performance liquid chromatography-mass spectrometry. Anal Chim Acta 691: 68-75, 2011

16. Taylor LA, Arends J, Hodina AK, Unger C and Massing U: Plasma lyso-phosphatidylcholine concentration is decreased in cancer patients with weight loss and activated inflammatory status. Lipids Health Dis 6: 17, 2007.

17. Yan JJ, Jung JS, Lee JE, Lee J, Huh SO, Kim HS, Jung KC, Cho JY, Nam JS, Suh HW, et al: Therapeutic effects of lysophosphatidylcholine in experimental sepsis. Nat Med 10: 161-167, 2004.

This work is licensed under a Creative Commons Attribution-NonCommercial-NoDerivatives 4.0 International (CC BY-NC-ND 4.0) License. 